**TITLE**

ASP Conference Series, Vol. **VOLUME**, **PUBLICATION YEAR **

**EDITORS ${ }^{* *}$

\title{
Scalar field models for an accelerating universe
}

\author{
Varun Sahni \\ Inter-University Centre for Astronomy \& Astrophysics, Post Bag 4, \\ Pune 411007, India
}

\begin{abstract}
I describe a new class of quintessence+CDM models in which late time scalar field oscillations can give rise to both quintessence and cold dark matter. Additionally, a versatile ansatz for the luminosity distance is used to reconstruct the quintessence equation of state in a model independent manner from observations of high redshift supernovae.
\end{abstract}

\section{A new model of quintessence and cold dark matter}

The supernova-based discovery that the universe may be accelerating can be explained within a general relativistic framework provided one speculates the presence of a matter component with negative pressure, the most famous example of which is the cosmological constant ' $\Lambda$ ' (Perlmutter et al. 1998,1999; Riess et al. 1999). $\Lambda$ runs into formidable fine tuning problems since its value must be set $\sim 10^{123}$ times smaller than the energy density in the universe at the Planck time in order to ensure that $\Lambda$ dominates the total energy density at precisely the present cosmological epoch. This involves a fine tuning of one part in $10^{123}$ at the Planck scale or one part in $10^{53}$ at the Electroweak scale.

One way around this difficulty is to make $\Lambda$ time-dependent, perhaps by using scalar field models which successfully generate a time-dependent $\Lambda$-term during an early Inflationary epoch. In this context the exponential potential provides an interesting illustration, since the density in the $\phi$-field tracks the background matter/radiation density when the latter is cosmologically dominant (Ratra \& Peebles 1988, Wetterich 1988, Ferreira \& Joyce 1997):

$$
\frac{\rho_{\phi}}{\rho_{B}+\rho_{\phi}}=\frac{3\left(1+w_{B}\right)}{p^{2} \lambda^{2}} \ll 1
$$

$\left(w_{B}=0,1 / 3\right.$ respectively for dust, radiation). This behaviour allows $\rho_{\phi}$ to be fairly large initially. Based on this property we introduce a new class of cosmological models which can describe both a time-dependent $\Lambda$-term (quintessence) and cold dark matter (CDM) within the unified framework of the class of potentials (Sahni \& Wang 2000)

$$
V(\phi)=V_{0}(\cosh \lambda \phi-1)^{p} .
$$

$V(\phi)$ has asymptotic forms:

$$
\begin{aligned}
& V(\phi) \simeq \tilde{V}_{0} e^{-p \lambda \phi} \text { for }|\lambda \phi| \gg 1(\phi<0), \\
& V(\phi) \simeq \tilde{V}_{0}(\lambda \phi)^{2 p} \text { for }|\lambda \phi| \ll 1 \\
& 1
\end{aligned}
$$


where $\tilde{V}_{0}=V_{0} / 2^{p}$. The exponential form of $V(\phi)$ guarantees that the scalar field equation of state mimics background matter at early times so that $w_{\phi} \simeq w_{B}$. At late times oscillations of $\phi$ lead to a mean equation of state

$$
\left\langle w_{\phi}\right\rangle=\left\langle\frac{\frac{1}{2} \dot{\phi}^{2}-V(\phi)}{\frac{1}{2} \dot{\phi}^{2}+V(\phi)}\right\rangle=\frac{p-1}{p+1},
$$

resulting in cold dark matter with $\left\langle w_{\phi}\right\rangle \simeq 0$ if $p=1$, or in quintessence with $\left\langle w_{\phi}\right\rangle \leq-1 / 3$ if $p \leq 1 / 2$. We therefore have before us the attractive possibility of describing CDM and quintessence in a common framework by the potential

$$
V(\phi, \psi)=V_{\phi}\left(\cosh \lambda_{\phi} \phi-1\right)^{p_{\phi}}+V_{\psi}\left(\cosh \lambda_{\psi} \psi-1\right)^{p_{\psi}}
$$

where $p_{\psi}=1$ in the case of CDM and $p_{\phi} \leq 0.5$ in the case of quintessence. In figure 1 we show a working example of this model which agrees well with observations of high redshift supernovae and does not suffer from the fine tuning problem faced by $\Lambda$, since $\rho_{\phi}$ can be fairly large initially. We should add that most models of quintessence usually work under the assumption that the three matter fields: baryons, CDM \& quintessence need not be related in any fundamental way and might even have different physical origins. If this is indeed the case then it remains somewhat of a mystery as to why $\Omega_{\phi}, \Omega_{m}$, (and possibly $\Omega_{b}$ ) have comparable magnitudes at the present time. By combining quintessence and CDM within a single class of potentials we make a small step in answering this question by showing that unified models of quintessence and CDM are conceivable (Sahni \& Wang 2000).

An intriguing property of cold dark matter based on (6) is that it can have a large Jeans length which leads to suppression (frustration) of clustering on kiloparsec scales. Frustrated Cold Dark Matter (FCDM) redresses certain shortcomings of the standard CDM scenario and might provide a natural explanation for the dearth of dwarf galaxies seen in our local neighborhood (Sahni \& Wang 2000).

Other quintessence potentials include $V(\phi) \propto \phi^{-\alpha}$ (Ratra \& Peebles 1988), $V(\phi) \propto e^{\beta \phi^{2}} \phi^{-\alpha}\left(\right.$ Brax \& Martin 2000) and $V(\phi) \propto \sinh ^{-2 p}\left(\phi+\phi_{0}\right)($ Sahni \& Starobinsky 2000). The latter describes quintessence which maintains a constant equation of state $w=-(1+p)^{-1}$ throughout the matter dominated epoch and later, during acceleration.

\section{Reconstructing quintessence from supernova observations}

Although a large class of scalar potentials can describe a time dependent $\Lambda$ term, no unique potential has so far emerged from a consideration of high energy physics theories such as supergravity or M-theory. (The situation in many respects resembles that faced by the Inflationary scenario, for a review see Sahni \& Starobinsky 2000.) It is therefore meaningful to try and reconstruct $V(\phi)$ directly from observations in a model independent manner. This is easy to do if one notes that, in a flat FRW universe, the luminosity distance determines the Hubble parameter uniquely (Starobinsky 1998, Saini et al. 2000)

$$
H(z) \equiv \frac{\dot{a}}{a}=\left[\frac{d}{d z}\left(\frac{D_{L}(z)}{1+z}\right)\right]^{-1}
$$




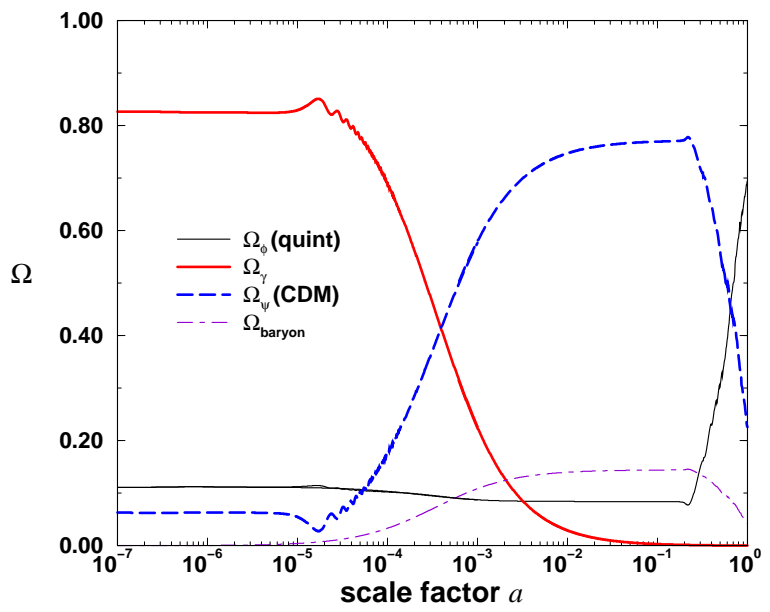

Figure 1. The evolution of the dimensionless density parameter for the CDM field $\Omega_{\psi}$ (dashed line) and quintessence field $\Omega_{\phi}$ (thin solid line). Baryon (dash-dotted line) and radiation densities (thick solid line) are also shown. For more details see Sahni and Wang (2000).

The Einstein equations can be written in the suggestive form

$$
\begin{aligned}
\frac{8 \pi G}{3 H_{0}^{2}} V(x) & =\frac{H^{2}}{H_{0}^{2}}-\frac{x}{6 H_{0}^{2}} \frac{d H^{2}}{d x}-\frac{1}{2} \Omega_{\mathrm{M}} x^{3}, \\
\frac{8 \pi G}{3 H_{0}^{2}}\left(\frac{d \phi}{d x}\right)^{2} & =\frac{2}{3 H_{0}^{2} x} \frac{d \ln H}{d x}-\frac{\Omega_{\mathrm{M}} x}{H^{2}},
\end{aligned}
$$

where $x \equiv 1+z$. Thus knowing $D_{L}$ we can determine both $H(z)$ and $d H(z) / d z$, and hence $V(\phi)$. The cosmic equation of state can also be reconstructed from $D_{L}$ since

$$
w_{\phi}(x) \equiv \frac{p}{\rho}=\frac{(2 x / 3) d \ln H / d x-1}{1-\left(H_{0}^{2} / H^{2}\right) \Omega_{\mathrm{M}} x^{3}} .
$$

In order to apply our method to observations we use the following rational ansatz for the luminosity distance

$$
\frac{D_{L}}{x} \equiv \frac{2}{H_{0}}\left[\frac{x-\alpha \sqrt{x}-1+\alpha}{\beta x+\gamma \sqrt{x}+2-\alpha-\beta-\gamma}\right]
$$

where $\alpha, \beta$ and $\gamma$ are fitting parameters. This function reproduces the exact analytical form of $D_{L}$ when $\Omega_{\phi}=0, \Omega_{\mathrm{M}}=1$ and when $\Omega_{\phi}=1, \Omega_{\mathrm{M}}=0$. It also has the correct asymptotic behaviour $H(z) / H_{0} \rightarrow 1$ for $z \rightarrow 0$, and $H(z) / H_{0} \rightarrow$ 


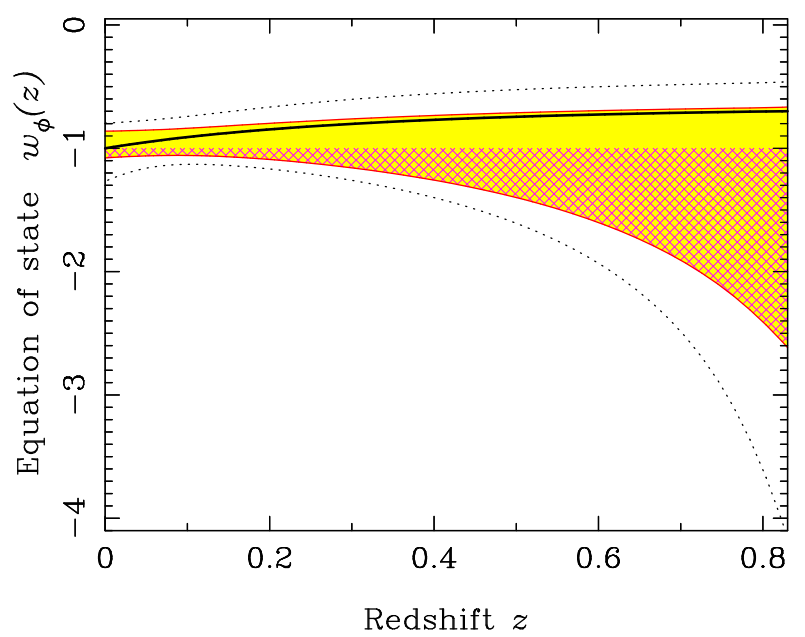

Figure 2. The equation of state parameter $w_{\phi}(z)=p_{\phi} / \rho_{\phi}$ as a function of redshift. The solid line corresponds to the best-fit values of the parameters. The shaded area covers the range of $68 \%$ errors, and the dotted lines the range of $90 \%$ errors. The hatched area represents the region $w_{\phi} \leq-1$, which is disallowed for a minimally coupled scalar field (from Saini et al. 2000).

$(1+z)^{3 / 2}$ for $z \gg 1$. Applying a maximum likelihood technique to $D_{L}$ given by (11) and $D_{L}^{o b s}$ obtained from observations of high redshift supernovae, we can reconstruct $H(z), V(\phi)$ and $w_{\phi}(z)$. Our results for $w_{\phi}$ shown in fig. 2 indicate some evidence of possible evolution in $w_{\phi}$ with $-1 \leq w_{\phi} \lesssim-0.80$ preferred at the present epoch, and $-1 \leq w_{\phi} \lesssim-0.46$ at $z=0.83$, the farthest $\mathrm{SN}$ in the sample (both at $90 \% \mathrm{CL}$ ). However, a cosmological constant with $w=-1$ is also consistent with the data.

Acknowledgments. The results presented in this talk were obtained in collaboration with Somak Raychaudhury, Tarun Saini, Alexei Starobinsky and Limin Wang whom I would like to thank for many enjoyable discussions.

\section{References}

Brax, P. and Martin, J. 2000, Phys. Rev. D, 61, 103502.

Ferreira, P.G. \& Joyce, M. 1997, Phys. Rev. Lett. 79, 4740.

Perlmutter, S.J. et al., 1998, Nature 391, 51.

Perlmutter, S.J. et al., 1999, ApJ, 517, 565.

Ratra, B. \& Peebles, P.J.E. 1988, Phys. Rev. D, 37, 3406.

Riess, A.G. et al., 1998, Astron. J. 116, 1009.

Sahni,V. \& Starobinsky, A.A. 2000, Int. J. Mod. Phys. D9, 373.

Sahni,V. \& Wang, L. 2000, Phys. Rev. D, (in press); astro-ph/9910097.

Saini, S., Raychaudhury, S., Sahni, V. \& Starobinsky, A.A. 2000, Phys. Rev. Lett. 85, 1162 .

Starobinsky, A.A. 1998, JETP Lett., 68, 757.

Wetterich, C. 1988, Nuclear Physics B 302, 668. 